\title{
Estudo radiológico do valor angular da cifose torácica em adolescentes
}

\author{
Radiological study of the angular value of thoracic kyphosis in \\ adolescents
}

\author{
Estudio radiológico del valor angular de la cifosis torácica en \\ adolescentes
}

\author{
Juan Javier Moreira Moreira' \\ Andres Edgar Rodriguez Fuentes ${ }^{2}$ \\ Paulo Henrique De Castro Corrêa ${ }^{1}$
}

\section{RESUMO}

Objetivo: determinar a diferença dos valores angulares da cifose torácica utilizando como vértebra terminal cranial diferentes níveis (T2 a T5). Métodos: foram avaliadas radiografias em perfil de cem adolescentes voluntários saudáveis da Escola Industrial do Serviço Social da Indústria (SESI) de Ribeirão Preto (SP), com prévia autorização dos pais ou responsáveis. Foram excluídas as radiografias de dez indivíduos por falhas na qualidade. Os parâmetros avaliados foram: mensuração da cifose torácica pelo método de Cobb, utilizando T2, T3, T4 ou T5 como vértebra terminal cranial e T12 como vértebra terminal caudal. Resultados: foram avaliados 90 indivíduos (46 do sexo masculino e 44 do feminino), com idade variando de 13 a 15 anos (média 14 \pm 6 ). O valor angular da cifose torácica nos diferentes níveis variou entre $45^{\circ}$ (T2-T12) e $35^{\circ}$ (T5-T12) no sexo masculino, e valor angular entre $43^{\circ}$ (T2-T12) e $30^{\circ}$ (T5-T12) no sexo feminino. Conclusão: foi observada diferença constante de aproxima-

\begin{abstract}
Objective: to determine the difference of the thoracic kyphosis angular values using different levels (T2 a T5) as a terminal cranial vertebra. Methods: sagittal radiographies of one hundred healthy adolescent volunteers, who study at Escola Industrial do Serviço Social da Indústria (SESI) in Ribeirão Preto SP), were evaluated the sagittal radiographies of one hundred health volunteers adolescent, that studies at Escola Industrial do SESI in Ribeirão Preto (SP), with parents consent. Ten adolescents were excluded because of flaws in the quality. The studied parameters were: the measurement of thoracic kyphosis by the Cobb method, using T2, T3, T4, T5 as a terminal proximal vertebra and T12 as a distal final vertebra. Results: Ninety individuals (46 men and 44 women), aged from 13 to 15 (average of $14 \pm 6$ ), were evaluated. The angular value of thoracic kyphosis in the different levels varied from $46^{\circ}$ (T2 - T12) to $35^{\circ}$ (T5 T12) in men, and from $44^{\circ}$ (T2- T12) to $30^{\circ}$ (T5 - T12) in women. Conclusion: A constant difference of approximately
\end{abstract}

\section{RESUMEN}

Objetivo: determinar la diferencia de los valores angulares de la cifosis torácica usando como vértebra terminal craneal, diferentes niveles (T2 a T5). Métodos: fueron evaluadas radiografias en perfil de cien adolescentes voluntarios saludables de la Escola Industrial do Serviço Social da Indústria (SESI) de Ribeirão Preto (SP), con previa autorización de sus padres o responsables. Fueron excluidas radiografias de diez individuos por fallas de resolución. Los parámetros evaluados fueron: la medida de la cifosis torácica por el método de Cobb, usando T2,T3,T4 y T5 como vértebra terminal craneal y T12 como vértebra terminal caudal. Resultados: fueron evaluados 90 individuos (46 hombres y 44 mujeres), con edades que varían de 13 a 15 años (media 14 46 ). El valor angular de la cifosis torácica en los diferentes niveles fue de $45^{\circ}$ (T2-T12) y $35^{\circ}$ (T5T12) en el sexo masculino, $y$ valor angular de 43 (T2-T12) y $30^{\circ}$ (T5-T12) en el sexo femenino. Conclusión: fue observada una diferencia constante de aproximadamente $5^{\circ}$ cuando los va-

\footnotetext{
Trabalho realizado no Serviço de Ortopedia e Traumatologia da Santa Casa de Ribeirão Preto - Ribeirão Preto (SP), Brasil.

'Médico Ortopedista; Assistente do Grupo de Coluna Vertebral do Serviço de Ortopedia e Traumatologia da Santa Casa de Ribeirão Preto - Ribeirão Preto (SP), Brasil.

${ }^{2}$ Doutor; Professor do Departamento de Biomecânica, Medicina e Reabilitação do Aparelho Locomotor da Faculdade de Medicina de Ribeirão Preto da Universidade de São Paulo - USP; Chefe do Serviço de Ortopedia e Traumatologia da Santa Casa de Ribeirão Preto - Ribeirão Preto (SP), Brasil.
} 
damente $5^{\circ}$ quando comparados os valores angulares da cifose torácica utilizando diferentes níveis (T2 a T5) como vértebra terminal cranial. $5^{\circ}$ was observed when comparing the angular values of thoracic kyphosis using different levels (T2 - T5) as a terminal cranial vertebra. lores angulares de la cifosis torácica fueron comparados, usando diferentes niveles (T2 a T5) como vértebra terminal craneal.
DESCRITORES: Vértebras torácicas; Curvaturas da coluna vertebral; Cifose/ radiografia; Avaliação; Adolescente
KEYWORDS: Thoracic vertebrae; Spinal curvatures; Kyphosis/ radiography; Evaluation; Adolescent
DESCRIPTORES: Vértebras torácicas; Curvaturas de la columna vertebral; Cifosis/ radiografía; Evaluación; Adolescente

\section{INTRODUÇÃO}

A coluna vertebral é uma estrutura mecânica complexa que sustenta o homem bípede e ereto. Vista no plano frontal é retilínea, e no plano sagital apresenta curvas fisiológicas importantes do ponto de vista biomecânico.

No período intrauterino, a coluna apresenta uma única curva de concavidade anterior. Após o nascimento, no decorrer do desenvolvimento do indivíduo, com a sustentação da cabeça, desenvolve-se uma curva cervical de convexidade anterior (lordose cervical). Quando começa a sentar ou engatinhar, desenvolve-se uma curva na região lombar de convexidade anterior, a lordose lombar. Finalmente, a curva inicial ao nível torácico que apresentava ao nascer se faz mais evidente, ou melhor, aumenta levemente, desenvolvendo-se a cifose torácica, que se completa com a inclinação da bacia que o indivíduo adota quando fica em pé ou deambula. Assim, temos a quarta curva fisiológica, que é a cifose sacrococcígea ${ }^{1}$.

De acordo com White e Panjabi ${ }^{2}$, as curvas fisiológicas da coluna vertebral têm substratos anatômicos importantes para sua presença. São elas que fornecem maior flexibilidade à coluna vertebral, aumentam sua capacidade de choque, mantêm uma adequada estabilidade estrutural e protegem a medula espinhal das forças deformantes ou movimentos bruscos.

No passado, as atenções foram direcionadas para a avaliação e tratamento das curvas no plano coronal, subestimando a importância do sagital. A nosso ver, isso ocorreu devido à falta de claros limites anatômicos para sua mensuração. Existe ainda controvérsia no que diz respeito aos limites aceitos como normais para as curvas fisiológicas ${ }^{3}$.

Diversos métodos de tratamento têm sido indicados para prevenir ou corrigir deformidade no plano sagital, porém, não existe consenso acerca das indicações de tratamento das hipercifoses torácicas e de uma melhor compreensão da história natural dessa deformidade.

É importante ressaltar que o aumento na curvatura torácica é uma das alterações posturais mais frequentes, própria de indivíduos adultos, secundária a processos degenerativos e agravada nas fraturas por osteoporose ${ }^{4}$. É também associada a patologias (Scheuermann, neurofibromatose etc.) em indivíduos mais jovens, podendo deslocar o centro de gravidade anteriormente, aumentando a instabilidade postural e levando à maior suscetibilidade a quedas por perda do balanço sagital. Pode causar dor instabilidade, deformidade cosmética, paraplegia e alterações cardiopulmonares ${ }^{5}$.

A avaliação anatômica e biomecânica da coluna torácica necessita de uma análise quantitativa das características dessa curva no plano sagital ${ }^{6}$.

Numerosos estudos reportaram cifoses com variações de $20^{\circ}$ a $40^{\circ}$ como razoavelmente normais, avaliadas por diferentes métodos de mensuração ${ }^{7}$.

Esse fato motivou a análise de radiografias em indivíduos normais, com o objetivo de estudar o valor angular da cifose torácica pelo método de $\mathrm{Cobb}^{8}$ e determinar os valores quando medidos em diferentes níveis.

\section{MÉTODOS}

O estudo foi conduzido no Serviço de Ortopedia e Traumatologia da Santa Casa de Ribeirão Preto (SP) em 100 adolescentes voluntários, estudantes da Escola Industrial do Serviço Social da Indústria (SESI), de diversas classes socioeconômicas da região, com autorização prévia dos pais ou responsáveis.

Foram excluídos dez indivíduos com evidência de anormalidades na coluna, bacia ou membros inferiores identificados no exame físico, tomando como parâmetros alinhamento de ombros, bacia e processos espinhos. $\mathrm{Na}$ tentativa de detectar a presença de escoliose, complementada pela inspeção lateral com os braços em flexão de $90^{\circ}$, observamos o aspecto estético e a presença de giba identificada pelo teste de Adams (Figuras 1 e 2).

Outro critério de exclusão foi a pobre definição das margens dos corpos vertebrais no exame radiológico de T2 a T12, totalizando 90 voluntários, 46 do sexo masculino e 44 do feminino, com idade que variou de 13 a 15 anos (média de $14 \pm 6$ ).

Foram realizadas radiografias da coluna torácica de todos os indivíduos em perfil ortostático com os braços a $90^{\circ}$ (Figura 1). Os filmes radiográficos utilizados foram de 30 x $40 \mathrm{~cm}$, posicionados a uma distância constante de 1 metro do foco e uma amplificação de $\pm 30 \%$. O equipamento era do tipo Multix ${ }^{\circledR}$, da marca Siemens, com um tubo de 


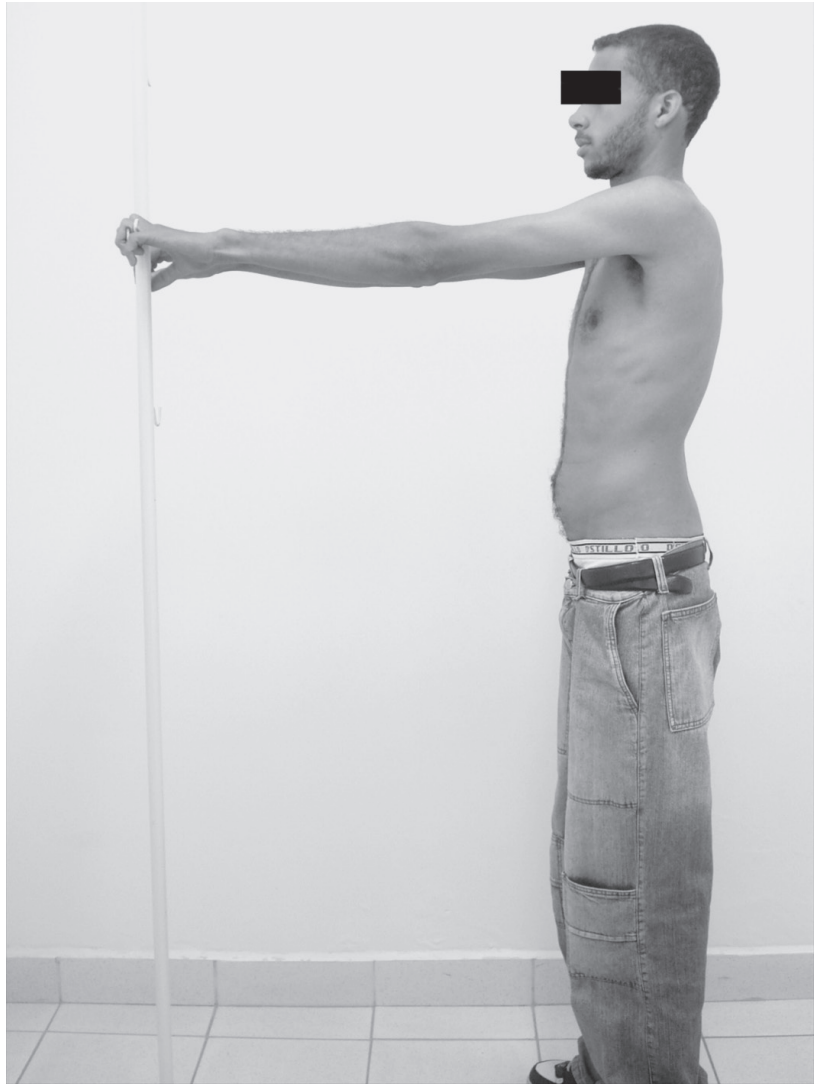

Figura 1

Posição para exame físico e radiológico.

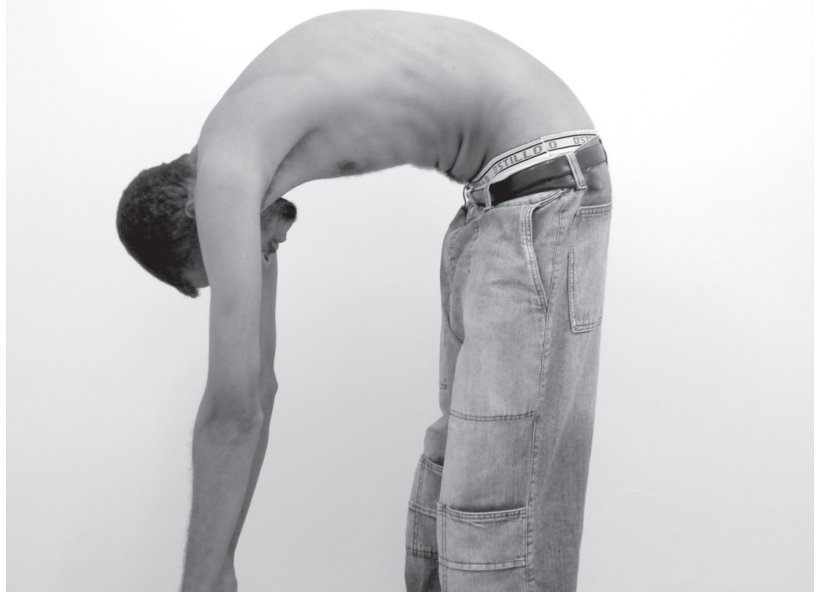

Figura 2

Teste de Adams.

Raios X, gerador Optamatic de $1.000 \mathrm{MA}, 150$ Kvolt e 100 Kw e a coraza Pantix PH 125/100 com P 135/30/50.

Foi mensurada a curva torácica por meio do método de $\mathrm{Cobb}^{8}$, utilizando-se o platô proximal de T2, T3, T4 e T5 como limite cranial, e como limite caudal o platô distal de T12 (Figura 3). Foram calculadas as médias, e os resultados foram comparados por sexo.

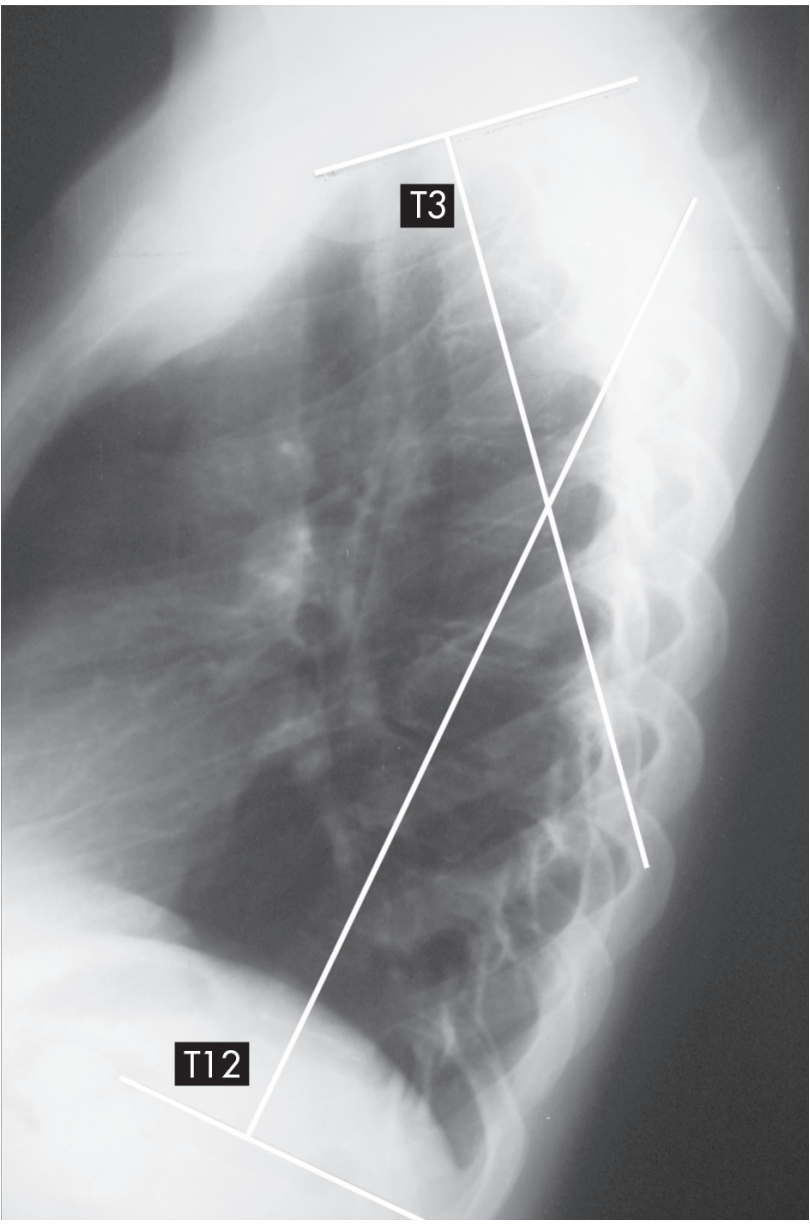

Figura 3

Mensuração da curva torácica pelo método de Cobb.

\section{RESULTADOS}

A Tabela 1 mostra os valores mínimo, máximo e a média para cada nível medido por sexo.

$\mathrm{O}$ valor angular da cifose torácica tem uma média que aumenta aproximadamente $5^{\circ}$ em cada nível à medida que utilizamos níveis mais superiores para a sua mensuração (Figura 4).

TABELA 1 - Observação das médias em relação aos níveis

Masculino Feminino

\begin{tabular}{|c|c|c|c|c|c|c|}
\hline & Mínimo & láximc & Média & Mínimo & Máximo & Média \\
\hline T2-Т12 & $20^{\circ}$ & $66^{\circ}$ & $45^{\circ}$ & $12^{\circ}$ & $58^{\circ}$ & $43^{\circ}$ \\
\hline T3-Т12 & $22^{\circ}$ & $62^{\circ}$ & $43^{\circ}$ & $11^{\circ}$ & $58^{\circ}$ & $39^{\circ}$ \\
\hline T4-Т1 2 & $22^{\circ}$ & $57^{\circ}$ & $39^{\circ}$ & $11^{\circ}$ & $51^{\circ}$ & $35^{\circ}$ \\
\hline T5-Т12 & $19^{\circ}$ & $50^{\circ}$ & $35^{\circ}$ & $17^{\circ}$ & $47^{\circ}$ & $30^{\circ}$ \\
\hline Média & & & $40,5^{\circ}$ & & & $36,75^{\circ}$ \\
\hline $\mathrm{O}^{\prime}$ & & & 4,43 & & & 5,5 \\
\hline
\end{tabular}

Comparados os resultados das médias por sexo, a cifose torácica no sexo masculino é cerca de $5^{\circ}$ maior que no feminino, independentemente do nível mensurado. 


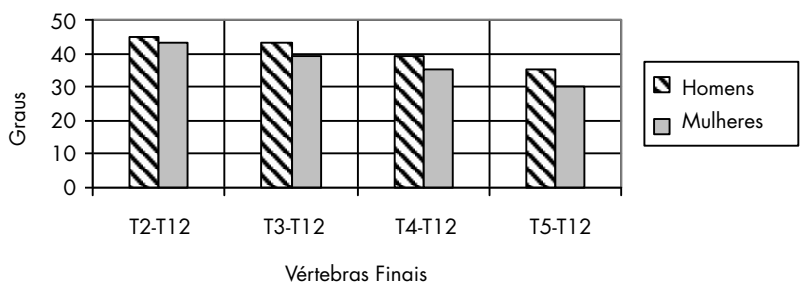

Figura 4

Comparação da média angular para cada nível por sexo.

\section{DISCUSSÃO}

A cifose torácica é relevante na conformação das curvaturas da coluna vertebral no perfil. Alterações angulares levam ao desequilíbrio sagital e a deformidades progressivas. Seu estudo é importante para melhorar a segurança no diagnóstico e o tratamento de cifose considerada anormal.

Winter et al. mencionam que a cifose é um problema específico, diferente da escoliose. Definem a cifose como um desvio da coluna no plano sagital além dos limites normais, não sendo claramente definido o que é normal ${ }^{9}$

A primeira tentativa para determinar a cifose torácica normal ou fisiológica foi realizada por Boseker em radiografias de 121 crianças sem patologias associadas. Estudou dois desvios-padrão da média como definição do normal $\left(20^{\circ}-50^{\circ}\right)$ sem diferença estatística quanto à idade e ao sexo. Neste estudo não foram apresentados claramente os níveis utilizados para medir a $\operatorname{cifose}^{10}$.

Sabe-se que há uma grande variabilidade na escolha da vértebra final cranial para determinar a cifose torácica ${ }^{11-16}$. Portanto, diferentes valores angulares são apresentados na literatura quando avaliados pelo método de Cobb, também utilizado no nosso estudo pela confiabilidade demonstrada em outros estudos ${ }^{8}$.

$\mathrm{Na}$ literatura, a cifose torácica considerada normal varia amplamente de $10^{\circ}$ a $40^{\circ}$, apresentando correlação positiva entre o aumento da idade e o aumento da cifose, sendo maior no sexo feminino quando comparado ao masculino $^{15}$ (dados não avaliados no nosso estudo por utilizarmos uma população mais jovem).

Axelgaard et al. ${ }^{17}$ estudaram 18 indivíduos, sendo 11 do sexo masculino, com média de idade de 14,6 anos, e 7 do sexo feminino, com média de idade de 13,8 anos. Desses indivíduos, 14 apresentavam doença de Scheuermann e 4 dorso curvo postural. A cifose nesses dois grupos foi de $42^{\circ}$ a $73^{\circ}$, com média de $57^{\circ}$, tomando como vértebras limites T3-T12, demonstrando uma diferença significativa quando comparados à nossa média no mesmo nível (T3T12), devido à inclusão de indivíduos com aumento da cifose. No nosso estudo, os voluntários foram avaliados pelo exame fisico, evitando a inclusão de indivíduos com hipercifose que pudessem interferir nos resultados.

Gelb et al. ${ }^{16}$ reconheceram que, embora a cifose comece em T1, essa vértebra, em geral, não pode ser visualizada em radiografias sagitais pela sobreposição do ombro. Essa observação levou a constatar que a cifose torácica superior de T1 a T5 em 100 adultos foi em média de 14 $\pm 8^{\circ}$. Adicionar esse número à cifose medida de T5 a T12 (média de $34^{\circ} \pm 11^{\circ}$ ) fornece uma estimativa razoável da cifose global. Nesse mesmo estudo, compararam-se as médias apresentadas por outros autores ${ }^{18,19}$, não demonstrando grande variabilidade da cifose quando comparado com os nossos resultados, principalmente no nível T5-T12.

Os resultados da média da cifose torácica nos diferentes níveis não apresentaram diferença significativa entre homens e mulheres quando comparados com outras séries publicadas quando se tratava de uma população menor de 40 anos. Após essa idade, a cifose tende a aumentar progressivamente, em especial no sexo feminino ${ }^{7}$.

Observamos que existem divergências tanto nos valores angulares da cifose torácica quanto na escolha da vértebra superior para a sua mensuração. Dessa forma, o presente estudo compara os valores angulares utilizando como parâmetros vários níveis (T2-T5) da cifose torácica em ambos os sexos, tentando esclarecer diferenças dos valores obtidos por outros autores. A faixa etária jovem estudada não permitiu conhecer a história natural da cifose torácica, como observado em outros estudos.

\section{CONCLUSÃO}

Foi possível concluir que os valores angulares da cifose torácica variam aproximadamente em média constante de $5^{\circ}$, quando comparamos cada nível (T2-T5) como vértebra final cranial. Há predominância aproximada de $5^{\circ} \mathrm{em}$ homens quando comparados com mulheres, independentemente do nível para a média de idade estudada.

\section{REFERÊNCIAS}

1. Gardner E. Anatomia. 38a ed. Rio de Janeiro: Guanabara-Koogan;1971.

2. White A, Panjabi MM. Clinical biomechanics of the spine. Philadelphia: JB Lippincott; 1978.

3. O'Rahilly R, Benson D. The development of the vertebral column. In: Bradford DS, Hensinger RN, editors. The pediatric spine. New York: Thieme, 1985. p. 3.
4. Vaillant J, Vuillerme N, Martigné P, Caillat-Miousse JL, Parisot J, Nougier V, et al. Balance, aging, and osteoporosis: effects of cognitive exercises combined with physiotherapy. Joint Bone Spine. 2006;73(4):414-8.
5. Lonner BS, Newton P, Betz R, Scharf C, O'Brien M, Sponseller $\mathrm{P}$, et al. Operative management of Scheuermann's kyphosis in 78 patients: radiographic outcomes, complications, and technique. Spine (Phila Pa 1976). 2007;32(24):2644-52. 
6. Singer KP, Jones TJ, Breidahl PD. A comparison of radiographic and computer-assisted measurements of thoracic and thoracoloumbar sagittal curvature. Skeletal Radiol. 1990;19(1):21-6.

7. Fon GT, Pitt MJ, Thies AC Jr. Thoracic kyphosis: range in normal subjects. AJR Am J Roentgenol. 1980;134(5):979-83.

8. Cobb JR. Outline for the studyof scoliosis. Am Acad Orthop Surg. 1984;9(1):65-70.

9. Winter RB, Hall JE. Kyphosis in childhood and adolescence. Spine (Phila Pa 1976).1978;3(4):285-308.

10. Boseker EH, Moe JH, Winter RB, Koop SE. Determination of "normal" thoracic kyphosis: a roentgenographic study of 121 "normal" children. J Pediatr Orthop. 2000;20(6):796-8.

11. Di Meglio A, Bonnel F. Growth of the spine. In: Raimondi AJ, Choux M, Di Rocco C, editors. The pediatric spine. New York: Springer-Verlag; 1989. Vol. 1. p. 39.

12. Herring JA. Tachdjian's pediatric orthopaedics. Dallas, Texas: Elsevier; 2008.
13. Kolessar DJ, Stollsteimer GT, Betz RR. The value of the measurement from T5 to T12 as a screening tool in detecting abnormal kyphosis. J Spinal Disord. 1996;9(3):220-2.

14. Goh S, Price RI, Leedman PJ, Singer KP. A comparison of three methods for measuring thoracic kyphosis: implications for clinical studies. Rheumatology (Oxford). 2000;39(3):310-5.

15. Macagno AE, O'Brien MF . Thoracic and thoracolumbar kyphosis in adults. Spine (Phila Pa 1976). 2006;31(19 Suppl):S161-70.

16. Gelb DE, Lenke LG, Bridwell KH, Blanke K, McEnery KW. An analysis of sagittal spinal alignment in 100 asymptomatic middle and older aged volunteers. Spine (Phila Pa 1976) 1995;20(12):1351-8.

17. Axelgaard J, Nordwall A, Brown JC. Correction of spinal curvatures by transcutaneous electrical muscle stimulation. Spine (Phila Pa 1976).1983;8(5):463-81.
18. Stagnara P, De Mauroy JC, Dran G, Gonon GP, Costanzo G, Dimnet J, et al. Reciprocal angulation of vertebral bodies in a sagittal plane: Approach to references for the evaluation of kyphosis and lordosis. Spine (Phila Pa 1976). 1982;7(4):335-42.

19. Bernhardt M, Bridwell KH Segmental analysis of the sagittal plane alignment of the normal thoracic and lumbar spines and thoracolumbar junction. Spine (Phila Pa 1976). 1989;14(7):171-21.

\section{Correspondência}

Juan Javier M Moreira

Serviço de Ortopedia e Traumatologia, Santa Casa

Avenida da Saudade, 456 - Campos Elíseos

CEP: 14085-000 - Ribeirão Preto (SP), Brasil

E-mail: jimmedical@hotmail.com; ortstacasarp@gmail.com 